\title{
Ablation Characteristics of Alumina and Zirconia Ceramics on Ultra-short Pulsed Laser Machining
}

\author{
Norbert Ackerl and Konrad Wegener \\ ETH Zurich, Institute of Machine Tools and Manufacturing (IWF), \\ Leonhardstrasse 21,8092 Zurich, Switzerland \\ E-mail: ackerln@ethz.ch
}

\begin{abstract}
Ultra-short pulsed laser manufacturing gives a unique route to ablate hard-to-machine materials and enables little thermal impact. A comparative study on an alumina-toughened zirconia (ATZ) composite and pure alumina is presented. Single- and multi-pulse ablation of $1030 \mathrm{~nm}$ and $515 \mathrm{~nm}$ wavelength with sub picosecond pulses reveal an abrupt increase of the material removal rate. Pockets with $5 \mu \mathrm{m}$ beam distance and 15 layers rotated by $23^{\circ}$ are generated to attain the multi-pulse ablation rate. The threshold fluence gives $0.1 \mathrm{~J} / \mathrm{cm}^{2}$ and $0.11 \mathrm{~J} / \mathrm{cm}^{2}$ for green wavelength and the ATZ and alumina, respectively. Single pulse ablation with $7 \mathrm{~J} / \mathrm{cm}^{2}$ energy density point to the sharp ablation limit and an electron microscopy assessment reveals a step-like behavior. Subsequent Raman spectroscopy studies prove the persistence of the tetragonal morphology of zirconia. These findings enable laser manufacturing of microstructures on ceramic specimen with a $2.5 \mathrm{D}$ and quasi-tangential strategy.
\end{abstract}

DOI: $10.2961 /$ jlmn.2019.02.0009

Keywords: laser machining, ablation, ultra-short pulsed laser, ceramic composites, micro structuring, quasi-tangential strategy, heat affected zone

\section{Introduction}

The development of novel metal oxide ceramics and compounds thereof gives the opportunity to choose the material properties for a certain application. Especially, these ceramics are usable in harsh working environments [1] and high temperature application with the need of superior mechanical properties [2,3]. Moreover, a very high corrosion resistance combined with low thermal conductivity makes ceramics an ideal candidate e.g. as a biomaterial [4] and potential successor of Ni-based single crystalline super alloys for turbine blades [5]. Following, technical ceramics have a broad use in different applications, but are conventionally hard-to-machine in the sintered state.

Laser manufacturing is one possible route to machine hard and ultra-hard materials in a defined way [6]. The use of ultra-short laser pulses enables the ablation of even high band gap materials. Although the laser wavelength is well below the gap, the high power density leads to multi-photon absorption, which generates charge carriers and makes defined laser machining possible [7]. The processes of laser cutting [8], drilling [9], and ablation [10] are reported in literature. The use of ceramics for bio-application and, especially, implants is a strongly growing market. Titanium implants have been the state-of-the-art material for the last two decades; however, structural ceramics have some unique properties. The mechanical properties are tunable, corrosion resistance is high, no allergic reactions are known, and from an esthetic perspective, white material is preferred in case of visible dental implants. Laser processing has therefore different future applications in the bio-medical industry. Recently, laser manufacturing of ceramic dental implants in rapid-prototyping way was demonstrated [11]. This gives the unique option to generate a precise geometry followed by surface structuring to enhance osseointegration [12]. Another application concerning surface modification is the structuring of ceramic crowns to increase the bonding strength and adhesion to the filling cement [13].

This contribution discusses the ablation characteristics of two ceramics - alumina and an alumina-toughened zirconia (ATZ) compound in the yttria stabilized tetragonal phase. A parameter study in the single- and multi-pulse regime points to a sharp onset of ablation and a high possible material removal rate. The parameter study serves as starting point to generate distinct micro- and nanostructures on the surface. Subsequently, the observed heat-affected zone in high-aspect ratio hole drilling and quasi-tangential ablation of rotational specimen is discussed qualitatively.

\section{Material and methods}

\subsection{Material}

The ceramics were ordered from Metoxit AG, Switzerland. A $99.8 \%$ purity alumina specimen in the $\alpha$ phase and an ATZ compound, specifically designed for highload application in aqueous environments, purchased. The stoichiometric composition of the alumina-toughened zirconia in mass percentage amounts to $76 \% \mathrm{ZrO}_{2}, 20 \%$ $\mathrm{Al}_{2} \mathrm{O}_{3}$, and $4 \% \mathrm{Y}_{2} \mathrm{O}_{3}$. In case of ceramics, $\mathrm{HfO}_{2}$ up to $6 \%$ is allowed as a byproduct to $\mathrm{ZrO}_{2}$, which is not listed by the suppliers. This leads to an unexpected peak in diffraction pattern and a hafnium signal in energy-dispersive $x$-ray spectroscopy assessments.

Both materials exhibit an indirect wide band gap depending on the phase. $\mathrm{ZrO}_{2}$ is monoclinic at room temperature with two transitions to tetragonal and cubic in the high-temperature regime. Yttria stabilizes the tetragonal phase, which is preferred with respect to mechanical 
properties. The gap in the tetragonal phase is about $5.5 \mathrm{eV}$, derived from theoretical computation [14] and is in good agreement to experimental measurements [15]. Alumina has a gap of about $8.7 \mathrm{eV}$ in the alpha phase [16] and $8.6 \mathrm{eV}$ in the gamma phase [17]. These gaps play a role in the electron generation after ultra-short pulsed excitation and influence the ablation behavior.

\subsection{Laser ablation testbed}

The setup is constituted by a combination of mechanical (XYZ) and optical axes (UV). The sketch in figure 1 reflects the configuration and Aerotech $\mathrm{GmbH}$ mechanical axes are combined with a galvo scanner from Scanlab. Aerotech controllers and the A3200 software synchronously control both systems. An Amphos laser source gives a wide

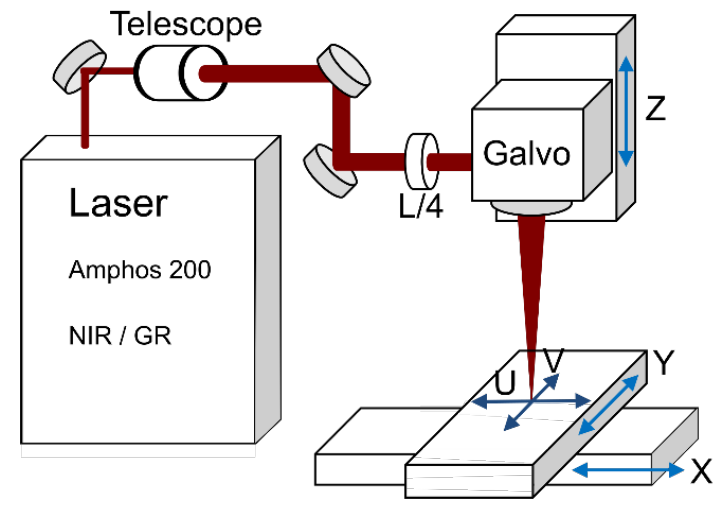

Fig. 1 Experimental testbed with five axes. The laser beam is modified by a telescope and the polarization controlled via wave plates. A galvo scanhead enables fast beam deflection on UV and the mechanical axes a precise positioning in $\mathrm{XY}$ and $\mathrm{Z}$.

parameter space in the near infrared (NIR) and green wavelength regime at $1030 \mathrm{~nm}$ and $515 \mathrm{~nm}$, respectively. The repetition rate can be tuned from $1 \mathrm{kHz}$ up to $40 \mathrm{MHz}$, which allows pulse picking and burst mode with a $25 \mathrm{~ns}$ spacing. The beam is modified in spatial dimension with a Galilean telescope and the polarization changed from linear to circular with a quarter-wave plate or tilted with a half-wave plate. This enables a defined polarization and focal spot diameter for ablation.

\subsection{Analysis methods}

The structures are inspected by optical microscopy and analyzed qualitatively by scanning electron microscopy (SEM). A Hitachi SU-70 with high resolution was used throughout this study and the non-conducting samples coated with $7 \mathrm{~nm}$ of amorphous carbon by a standard carbon coater unit. A confocal Leica DCM allows measuring the height and depth of the sample. Nevertheless, small lateral sized structures with high aspect ratio, like grooves and holes, cannot be resolved in detail due to multiple reflections. Raman spectroscopy of the ceramic ATZ compound allows determining the phase configuration. The spectra for comparison of the tetragonal, monoclinic, and cubic phase are reported in literature [18]. A Witec alpha 300R with green excitation wavelength at $532 \mathrm{~nm}$ gives a good signalto-noise ratio and sharp Raman peaks.

\section{Parameter study}

A study of the Alumina and ATZ with NIR and green wavelength has been carried out. Generally, a lower threshold fluence and higher ablation rate is expected for green laser radiation, considering the double photon energy compared to NIR. The absorption is in the non-linear regime and multiple photons needed to overcome the energy gap [19]. Therefore, a high power density should enhance the ablation process.

\subsection{Scanning strategy and slicing}

The experimental study was conducted on the introduced experimental configuration. In case of single pulse ablation, the repetition rate was reduced to $1 \mathrm{kHz}$ and at least 30 single pulse ablation craters generated by separating the pulses with the galvo scanner at $100 \mathrm{~mm} / \mathrm{s}$ feedrate.

The multi-pulse study and microstructures are generated by $2.5 \mathrm{D}$ layered orthogonal laser ablation. A computer aided manufacturing tool enables flexible laser manufacturing, where the designed geometry is imported and the laser paths and hatches are calculated [20]. Each adjacent layer is rotated by a prime number to minimize the error introduced by overlapping beam paths. The feedrate in conjunction with the laser repetition rate and focal diameter gives the pulse overlap. Following, the beam overlap is defined in the program and a sky writing length introduced to keep the ablation rate constant. The Aerotech setup enables a fast triggering and synchronized movement of all axes. However, for small structures spread over the whole objectives field of view, the controllers limit the maximal feedrate. These controllers are able to process a maximum of one command per microsecond. If more commands are necessary a buffer underrun occurs restricting the processing speed for specific cases [21].

\subsection{Material removal rate}

Pockets are ablated with 30 layers, a rotation of $23^{\circ}$ per layer, a $5 \mu \mathrm{m}$ beam distance, and a pulse overlap of $90 \%$. The average power and the wavelength are varied leading to different depths. Figure 2a shows the layer depth dependent on the fluence and wavelength for the two ceramics. If the volume removal rate is divided by the used average power, the specific material removal rate can be determined [22]. Moreover, an ablation efficiency can be calculated, assuming a logarithmic ablation law and a Gaussian distribution of the fluence. This leads to a maximum [23] with the optimal pulse energy of:

$$
\mathrm{E}_{P, o p t}=\frac{e^{2}}{2} \omega_{0}^{2} \pi F_{t h}
$$

The optimal fluence depends solely on the threshold fluence $F_{t h}$ and the focal beam radius $\omega_{0}$. Figure $2 \mathrm{~b}$ shows a modified specific material removal rate, where the layer depth is divided by the average power. If the same pocket geometry is generated with constant feedrate, repetition rate and hatch overlap the functionality $\dot{V} / P_{\text {avg }}$ can be reduced to the ablation depth $Z_{a b l}$. In case of green wavelength, this maximum at the optimal fluence of $0.7 \mathrm{Jcm}^{-2}$ for ATZ and $0.9 \mathrm{Jcm}^{-2}$ for alumina can be observed. However, NIR ablation solely revealed a kink and the maximum would be reached at higher fluence. 

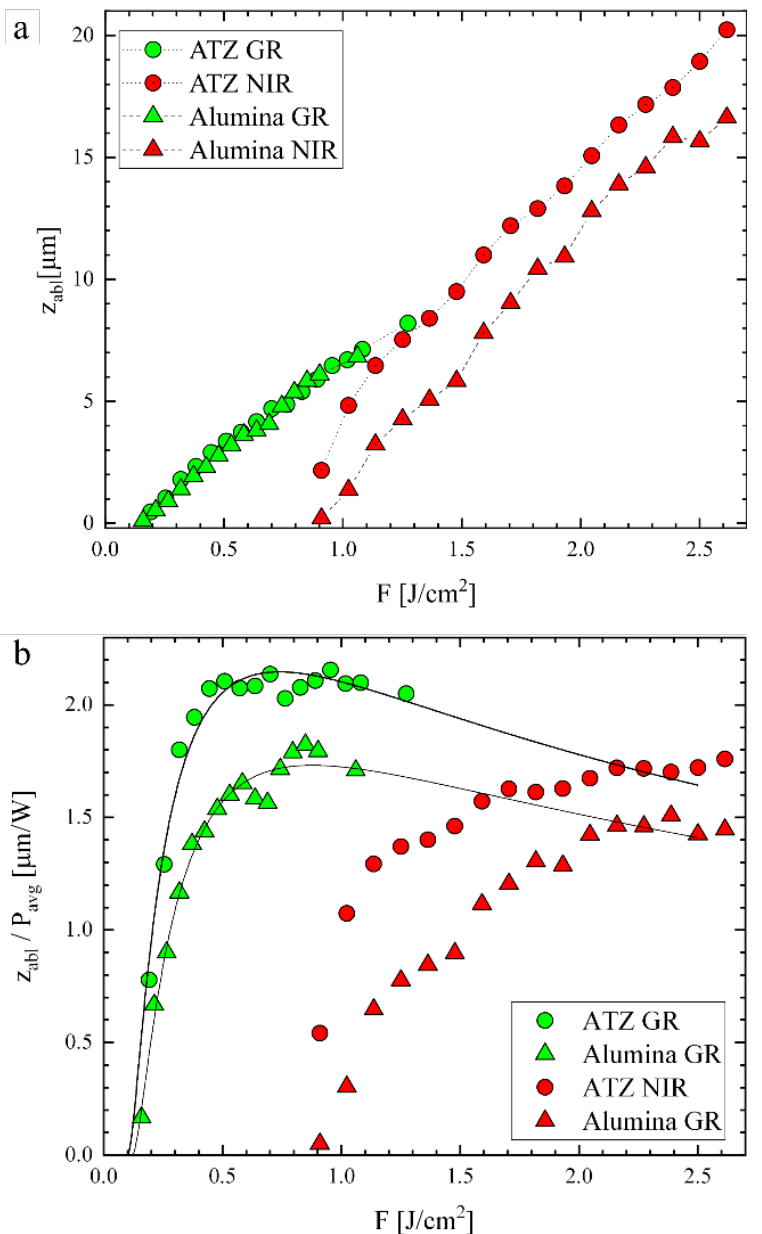

Fig. 2 Ablation study with NIR and green wavelength varying the fluence. The layer depth (a) increases with higher average power. In (b) the specific material removal rate reveals the optimal fluence regime for power efficient ablation with a curve fit in solid black after [23].

The absorption starts mainly at grain boundaries leading to ablation. Moreover, an impact of the plasma plume generated confinement could play a role and lead to the kink in the specific material removal rate [24]. For single pulse ablation, a sharp onset points to the threshold of generating electrons in the dielectric specimen. Hence, the power density necessary for excitation defines a small parameter window at the threshold value.

\subsection{Phase transformation}

A Raman study on ATZ after ultra-short pulsed laser ablation shows a negligible phase shift in the spectra. Figure 3 shows the attained spectra before and after laser ablation. The yellow bands point to the characteristic peaks for the

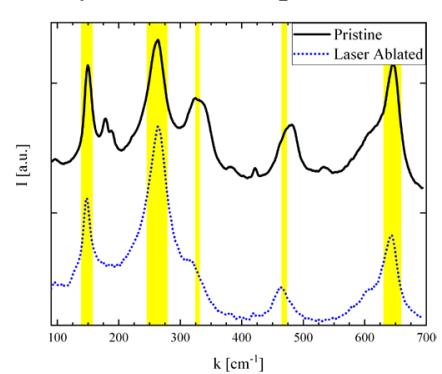

tetragonal phase. Clearly, in the pristine sample a double peak at $190 \mathrm{~cm}^{-1}$ and small peaks at $410 \mathrm{~cm}^{-1}$ and $520 \mathrm{~cm}^{-1}$ are visible. These peaks point to a small fraction of the monoclinic morphology in the specimen, which vanishes Fig. 3 Raman spectra of the ATZ after laser machining. ceramic before and after laser Nevertheless, cracks were ablation and yellow marked peaks. observed after certain processes at the surface, where no phase transition were detected by Raman spectroscopy.

\section{Experimental results and discussion \\ 4.1 Single laser pulse ablation of ATZ}

Low fluence green single pulse ablation revealed a sharp onset of ablation. The sample showed a glowing behavior due to multiple scattering of the incidence laser on the grain boundaries during processing. At the threshold fluence modification partially starts strongly depending on the material inhomogeneity. Figure 4 depicts a crater after ablation at $7 \mathrm{Jcm}^{-2}$, well above the threshold. Interestingly,

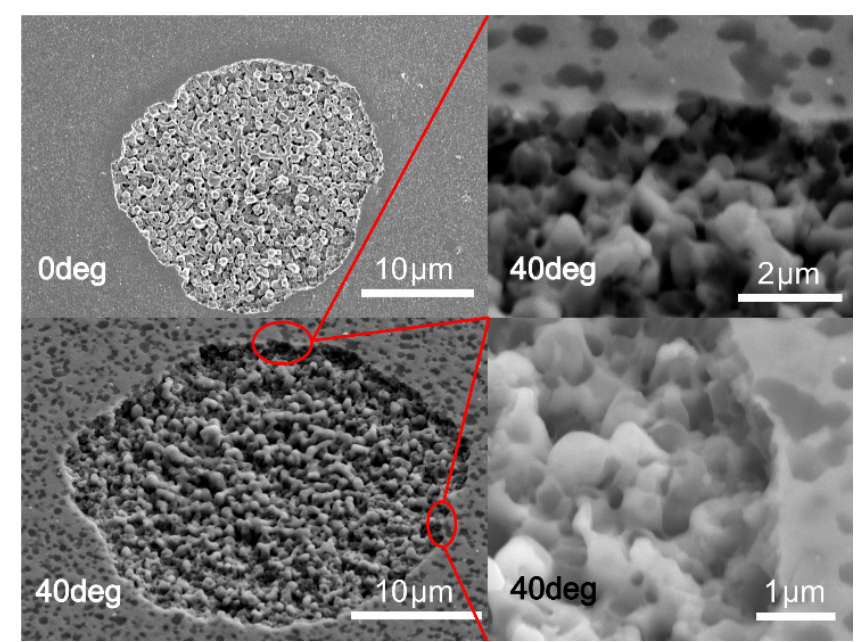

Fig. 4 Single pulse ablation on ATZ with $7 \mathrm{Jcm}^{-2}$ fluence leads to a sharp ablation edge, observable in the $40^{\circ}$ tilted SEM graphs.

the crater size does not change drastically with fluence like observed for metals, shown in figure $5 \mathrm{a}$ with a crater at $1 \mathrm{Jcm}^{-2}$ and in $5 \mathrm{~b}$ at $7 \mathrm{Jcm}^{-2}$. The diameter only increases from $20 \mu \mathrm{m}$ to $23 \mu \mathrm{m}$. However, even at moderate fluence small cracks are observed. This is qualitatively shown in figure $5 \mathrm{c}$ with a zoom from $5 \mathrm{a}$ and the small particle at the bottom in $5 \mathrm{~d}$. Still, in all cases the structure of the ATZ persists and even at the boundary of ablation no obvious structural change is observed and the black alumina particles persist, compare figure 4 and $5 \mathrm{~d}$.
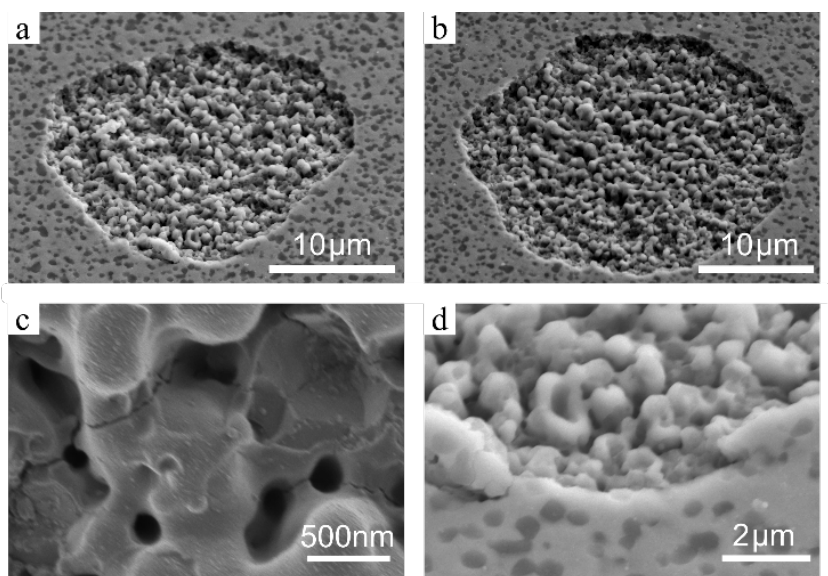

Fig. 5 Single pulse ablation at $1 \mathrm{Jcm}^{-2}$ in (a) and $7 \mathrm{Jcm}^{-2}$ (b). The figures (c) and (d) show a magnified version of (a) revealing small cracks in the centrum and a particle partially removed at the rim of the crater. 


\subsection{Radial micro structuring of ATZ ceramics}

With the parameters attained form section 3.2 distinct microstructures are introduced to the surface of the ATZ ceramic compound. Figure 6 gives an overview of grooves, cones and truncated pyramids. All structures are laser machined with a $2.5 D$ orthogonal strategy and the slicing carried out with $23^{\circ}$ rotation of each layer. In total 30 layers were removed from the material leading to $50 \mu \mathrm{m}$ of depth. The groove in figure $6 \mathrm{a}, \mathrm{b}$ shows a bridge region of below $5 \mu \mathrm{m}$ attained with a focal beam diameter of around $20 \mu \mathrm{m}$
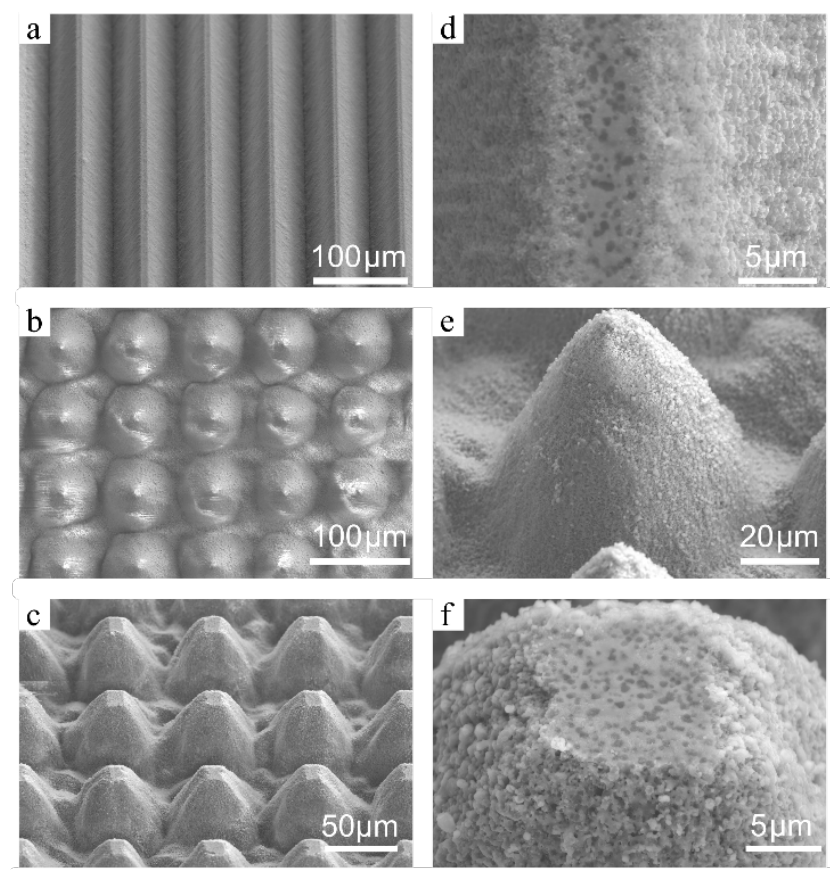

Fig. 6 Microstructures on the ATZ ceramics, the left column (a,b,c) is magnified and shown to the right $(\mathrm{d}, \mathrm{e}, \mathrm{f})$. Micro grooves $(\mathrm{a}, \mathrm{b})$, cones $(\mathrm{b}, \mathrm{e})$, and truncated pyramids $(\mathrm{c}, \mathrm{f})$ point to a flexible structuring routine.

and $0.2 \mathrm{Jcm}^{-2}$ fluence. In terms of processing time, a $5 \times 5 \mathrm{~mm}^{2}$ field of these structures takes $5 \mathrm{~min}$. However, the smaller the pitch of these structures the limits of the controller are more striking. All structures were limited to a feed rate of $250 \mathrm{~mm} / \mathrm{s}$ due to the controller speed restrictions.

\subsection{Micro drilling}

Laser machining gives the unique option for micro drilling in hard-to-machine materials, where conventional manufacturing techniques fail. An arrangement of $20 \mu \mathrm{m}$ holes with a distance of $70 \mu \mathrm{m}$ was generated on the ATZ ceramic. Figure 7 a depicts the drilled holes with a lateral shift of $35 \mu \mathrm{m}$ in both directions for a close packing. The magnified graph in figure $7 \mathrm{~b}$ shows the roundness of the holes with a typical taper angle leading to a conical shape. Figure $7 \mathrm{c}$ and $7 \mathrm{~d}$ reveal the holes at a tilting angle, where at the rim in figure $7 \mathrm{~d}$ black alumina particles point to a negligible HAZ. The used strategy was a circular laser hatch, where the centrum was parametrized to enable hole drilling in the whole field of view from the objective lens. A total of $200^{\prime} 000$ pulses are shot at the surface, while hatching the laser beam at $0.2 \mathrm{Jcm}^{-2}$ with $500 \mathrm{~mm} / \mathrm{s}$. This set of parameter leads to holes being the negative of the micro cones in figure 6 with a depth of $>50 \mu \mathrm{m}$.
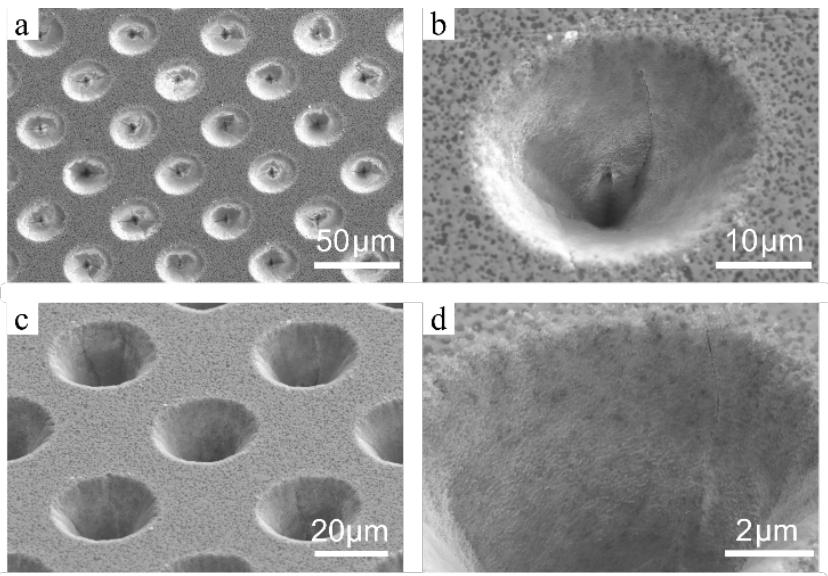

Fig. 7 Micro holes laser drilled in an ATZ ceramic with a circular laser hatch. Symmetric arrangement (a) of holes with $20 \mu \mathrm{m}$ diameter (b). In (c) the tilted graph reveals the sharp defined edge with magnification (c) showing the rim with alumina particles.

An extended parameter study for process scaling between $0.5 \mathrm{~W}$ to $2 \mathrm{~W}$ of the same process points to limitations. Figure 8 reveals the $\mathrm{HAZ}$ at $2 \mathrm{~W}$ average power and a fluence of $0.8 \mathrm{~J} / \mathrm{cm}^{2}$. Especially, the region seen in figure $8 \mathrm{~b}$ reveals cracks and the black alumina particles vanish. This dissolution could explain the generation of
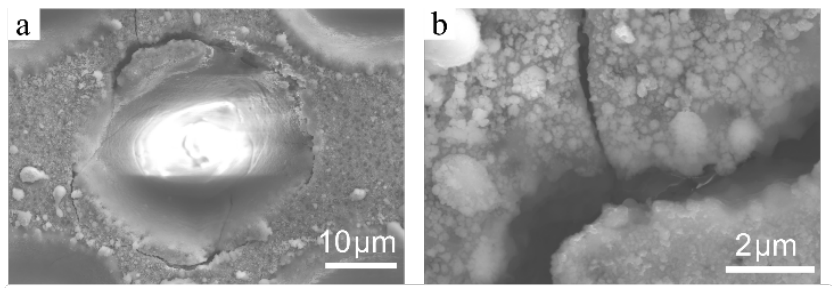

Fig. 8 Close up of a micro hole drilled at $0.8 \mathrm{Jcm}^{-2}$ with debris stuck on the surface (a). A magnification at the left top rim (b) shows a dissolved alumina phase and cracks.

cracks due to buildup of stress on the zirconia grain boundaries. Moreover, a mixing of zirconia and alumina could appear at high temperatures considering the eutectic temperature of $1800^{\circ} \mathrm{C}$ of the alumina-zirconia system.

\subsection{Quasi-tangential laser manufacturing}

A dental ceramic implant manufactured by quasitangential strategies serves as contrast to the observed HAZ occurring after micro drilling. Figure 9a shows a parameter study on a cylindrical specimen, which enables the laser manufacturing of complex $3 D$ geometries given in figure $9 \mathrm{~b}$. A detailed discussion on the manufacturing of ceramic
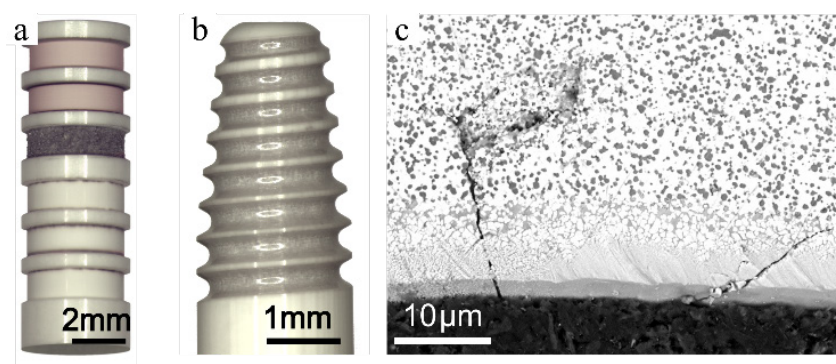

Fig. 9 Quasi-tangential parameter study on a rotational ATZ specimen (a) enabling the laser manufacturing of a dental implant (b). A closer look on the cross-section reveals a HAZ and cracks, similar to micro holes. 
dental implants is presented in [11], however, here we concentrate on the HAZ. Figure 9c shows the zone after quasi-tangential polishing in more detail and obviously, the spherical black alumina particles vanish near the surface. This seems to lead to the cracks observed in case of quasitangential laser processing, similar to the micro hole drilling in figure $8 \mathrm{~b}$. The process of drilling leads to glancing incidence condition on the flanks, if progressing deeper. Following, a part of the Gaussian laser beam is dissipated without material removal, which points to heating

Subsuming, a small parameter space points to distinct structuring of ceramic compounds with photon energies below the band gap and 1ps pulse duration. Even shorter pulses in the femtosecond regime could be beneficial and the higher power density at same pulse energy decrease the HAZ. Moreover, a laser source with ultra-short pulses and high photon energy, e.g. deep ultra-violet radiation, could lead to superior results on wide band gap materials [25]. The shorter wavelength would allow shrinking the minimal feature size and, following, the achievable pitch. Despite a possible smaller focal spot the Rayleigh length increases, which is beneficial for laser machining with an increase in the depth of focus.

\section{Conclusions and outlook}

An overview of the laser machining on two hard-tomachine materials was given. The ultra-short pulsed laser ablation characteristics of alumina and an aluminatoughened zirconia compound were discussed in detail. Following, a parameter study on single and multi-pulse ablation enabled the laser machining of structures that are more complex. Specifically, micro grooves, cones and truncated pyramids with highly defined geometry were presented. Micro hole drilling with an aspect ratio of 1:3 and an entrance diameter of $20 \mu \mathrm{m}$ pointed to a fast machining with a circular hatched laser beam. The process is sensitive to the energy density and a heat-affected zone was detected in the SEM graphs at moderate energy density. A comparison with a study on quasi-tangential processing points to the dissolution of alumina in a small region, which leads to cracks. This dissolution can be avoided by a careful choice of parameters with the drawback of longer processing times.

\section{Acknowledgments}

The authors would like to thank the Swiss National Science Foundation [SNSF $(\mathrm{CH})$ ] under the project "FuSSI Tools" (No. 169654) for financial support. N.A. wants to thank Johannes Gysel for his excellent work on quasi-tangential laser manufacturing of ceramic dental implants. N.A personally thanks Prof. Löffler, head of the LMPT at ETH Zurich, for access to the groups Hitachi SU-70 SEM.

\section{References}

[1] P. Kohorst, L. Borchers, J. Strempel, M. Stiesch, T. Hassel, F.-W. Bach, C. Hübsch: Acta Biomater., 8, (2012) 1213

[2] G. Magnani, A. Brillante: J. Eur. Ceram. Soc., 25, (2005) 3383

[3] F. Kern, H. Reveron, J. Chevalier, R. Gadow: J. Mech. Behav. Biomed. Mater., 90, (2019) 395.

[4] M. Fornabaio, H. Reveron, E. Adolfsson, L. Montanaro, J. Chevalier, P. Palmero: "Advances in Ceramic Biomaterials", ed. by P. Palermo, F. Cambier and E.D. Barra, (Elsevier, 2017) 355.

[5] Z. Fan, Y. Zhao, Q. Tan, N. Mo, M.-X. Zhang, M. Lu H. Huang: Acta Mater., 170, (2019) 24.

[6] A.N. Samant, N.B. Dahotre: J. Eur. Ceram. Soc., 29, (2009) 969.

[7] N.M. Bulgakova, I.M. Burakov, Y. Meshcheryakov, R Stoian: J. Laser Micro/Nanoeng., 2, (2007) 76.

[8] R.N. Oosterbeek, T. Ward, S. Ashforth, O. Bodley, A.E. Rodda, M.C. Simpson: Opt. Lasers Eng., 84, (2016) 105.

[9] H. Wang, H. Lin, C. Wang, L. Zheng, X. Hu: J. Eur. Ceram. Soc., 37, (2017) 1157.

[10] Y. Di Maio, J.P. Colombier, P. Cazottes, E. Audouard: Opt. Lasers Eng., 50, (2012) 1582.

[11] N. Ackerl, M. Warhanek, J. Gysel, K. Wegener: J. Eur. Ceram. Soc., 39, (2019) 1635.

[12] N. Ackerl, J. Gysel, M.G. Warhanek, K. Wegener: Proc. SPIE, Lasers Dent. XXV, (2019) 108570J1.

[13] N. Bärsch: J. Laser Micro/Nanoeng., 3, (2008) 78.

[14] F. Zandiehnadem, R.A. Murray, W.Y. Ching: Phys. B + C, 150, (1988) 19.

[15] H. Jiang, R.I. Gomez-Abal, P. Rinke, M. Scheffler: Phys. Rev. B, 81, (2010) 1.

[16] S. Ciraci, I.P. Batra: Phys. Rev. B, 28, (1983) 982-992.

[17] S.-D. Mo, Y.-N. Xu, W.-Y. Ching: J. Am. Ceram. Soc., 80, (2005) 1193.

[18] K. Byung-Kook, H. Jin-Woo, H. Kyoung R.: J. Mater. Sci. Lett., 16, (1997) 669.

[19] P. Boerner, M. Hajri, N. Ackerl, K. Wegener: J. Laser Appl., 31, (2019) 022202.

[20] N. Ackerl, M. Warhanek, J. Gysel, K. Wegener: Int. J. Adv. Manuf. Technol., 103, (2019) 1105.

[21] N. Ackerl, P. Boerner, K. Wegener: J. Laser Appl., 31, (2019) 022501.

[22] B. Neuenschwander, G.F. Bucher, C. Nussbaum, B. Joss, M. Muralt, U.W. Hunziker, P. Schuetz: Proc. SPIE, Laser Appl. Microelectron. Optoelectron. Manuf. XV, (2010) 75840R1.

[23] B. Neuenschwander, B. Jaeggi, M. Schmid, G. Hennig: Phys. Proc., 56, (2014) 1047.

[24] A. Palya, O.A. Ranjbar, Z. Lin, A.N. Volkov: Int. J. Heat Mass Transf., 132, (2019) 1029.

[25] J. Ihlemann, A. Scholl, H. Schmidt, B. Wolff-Rottke: Appl. Phys. A Mater. Sci. Process., 60, (1995) 411.

(Received: May 20, 2019, Accepted: August 26, 2019) 